\title{
Space-time singularities in Weyl manifolds
}

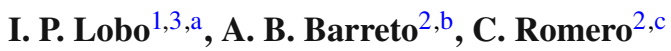 \\ ${ }^{1}$ CAPES Foundation, Ministry of Education of Brazil, Brasilia, Brazil \\ 2 Departamento de Física, Universidade Federal da Paraíba, C. Postal 5008, João Pessoa, PB 58051-970, Brazil \\ ${ }^{3}$ Dipartimento di Fisica, Sapienza Università di Roma, P.le Aldo Moro 5, 00185 Rome, Italy
}

Received: 13 August 2015 / Accepted: 8 September 2015 / Published online: 25 September 2015

(c) The Author(s) 2015. This article is published with open access at Springerlink.com

\begin{abstract}
We extend one of the Hawking-Penrose singularity theorems in general relativity to the case of some scalartensor gravity theories in which the scalar field has a geometrical character and space-time has the mathematical structure of a Weyl integrable space-time. We adopt an invariant formalism, so that the extended version of the theorem does not depend on a particular frame.
\end{abstract}

\section{Introduction}

Until the mid-1960s it was argued by some cosmologists that the presence of space-time singularities in general relativistic cosmological models was not an essential property of the model, being, in fact, a consequence of the high degree of symmetries of the distribution of matter assumed in these models [1]. Accordingly, it was believed that in more realistic situations these singularities would disappear. However, this scenario was to change drastically after a series of general mathematical results, namely, the so-called Hawking-Penrose singularity theorems, were proved [2-5]. As is widely known, these theorems use methods of global analysis to show that, under the assumption of the validity of general relativity and a reasonable physical behavior of matter, space-time singularities are general phenomena which occur in gravitational collapse and cosmology (such as the big bang) irrespective of the symmetry of the models.

The investigation of space-time singularities has touched on some deep philosophical issues and it is the view taken by many scientists that these issues seem to call for a conceptual revision of general relativity that at least take quantum mechanics into account. As far as cosmological singularities are concerned it seems that the current view held by most cosmologists is that general relativity must break down at

\footnotetext{
a e-mail: iarley.pereiralobo@icranet.org

b e-mail: adrianobraga@fisica.ufpb.br

c e-mail: cromero@ fisica.ufpb.br
}

times less than the Planck time. In fact, there has been a fair deal of work on classical and quantum new approaches to gravity in which mechanisms that naturally prevent the formation of a cosmological singularity are present. In fact, from the standpoint of quantum mechanics the mere existence of a cosmological singularity would be in contradiction with Heisenberg's uncertainty principle. In connection with this point, many efforts have been made to understand what could be called the quantum structure of space-time. Among the numerous attempts to make progress in this rather difficult issue, we would like to call attention for a recent proposal which argues that the quantum structure of space-time may even be related to the possibility of the space-time geometry possessing a non-Riemannian character [6]. On the other hand, non-Riemannian geometries have appeared in physics mainly as a way to modify Einstein's gravity while keeping the idea that the gravitational field is a manifestation of the space-time geometry $[7,8]$. As is well known, one of the earlier attempts in this direction was the Weyl unified field theory [9-16].

Concerning space-time singularities, we know that modified theories of gravity call for a new mathematical treatment of the problem, as the approach provided by Hawking and Penrose is suitable only for general relativity. It is our purpose here to pursue this question further in the light of some modified gravity theories formulated in a particular kind of space-time geometry, namely, the so-called Weyl integrable space-time (WIST) [17-29].

The present article is organized as follows. In Sect. 2, we outline the fundamental ideas of the geometry developed by Weyl, which underlies his unified field theory. We then proceed to briefly review the main features of Weyl's attempt to unify gravity and electrodynamics in a single geometric framework. In Sect. 3, we consider the extension of Raychaudhuri equation from a Riemannian setting to the case of a Weyl integrable space-time. This extension is invariant under Weyl transformations. In Sect. 4, we work out a gen- 
eralization of one of the Hawking-Penrose singularity theorems. Some applications of the formalism are given in Sect. 5 . These correspond to cosmological solutions of scalar-tensor theories framed in a Weylian space-time. We conclude with some remarks in Sect. 6.

\section{Weyl geometry}

Weyl geometry arises from the weakening of one of the postulates of the Riemannian geometry, similarly as non-Euclidean geometry was conveived after the fifth postulate of Euclidean geometry was relaxed. The postulate we are referring to is the so-called the Riemannian compatibility condition, which states the following.

Postulate 2.1 Let $M$ be a differentiable manifold endowed with an affine connection $\nabla$ and a metric tensor $g$. For any vector fields $U, V, W \in T(M)$, it is required that

$V[g(U, W)]=g\left(\nabla_{V} U, W\right)+g\left(U, \nabla_{V} W\right)$.

As is well known, this condition is equivalent to the requirement that the covariant derivative of the metric vanish, thereby implying that the length of a vector remain unaltered by parallel transport.

On the other hand, in Riemannian geometry, it will also be assumed that the connection $\nabla$ be torsionless (or symmetric), i.e., that for any $U, V \in T(M)$ the following condition holds:

$\nabla_{V} U-\nabla_{U} V=[V, U]$

From these two postulates we are led to the important LeviCivita theorem, which states that the affine connection is entirely determined from the metric [30]. In a certain sense, this theorem characterizes a Riemannian manifold.

In 1918 the mathematician Hermann Weyl generalized the geometry of Riemann by introducing the possibility of change in the length of a vector through parallel transport. To implement this idea Weyl conceived the following new compatibility condition.

Postulate 2.2 (Weyl) Let $M$ be a differentiable manifold endowed with an affine connection $\nabla$, a metric tensor $g$ and a one-form field $\sigma$, called a Weyl field. It is said that $\nabla$ is compatible (W-compatible) with $g$ if for any vector fields $U, V$, $W \in T(M)$, we have

$V[g(U, W)]=g\left(\nabla_{V} U, W\right)+g\left(U, \nabla_{V} W\right)+\sigma(V) g(U, W)$.

Clearly, this represents a generalization of the Riemannian compatibility condition. Naturally, if the one-form $\sigma$ vanishes, we reobtain (1). In this way, we have a generalized version of the Levi-Civita theorem given by the following proposition (see Ref. [31] for a proof):

Theorem 2.1 (Levi-Civita extended) Let $M$ be a differentiable manifold endowed with a metric $g$ and a differentiable one-form field $\sigma$ defined on $M$, then there exists one and only one affine connection $\nabla$ such that: ( $i) \nabla$ is torsionless; ( $i i$ ) $\nabla$ is $W$-compatible with $g$.

It follows that in a coordinate basis $\left\{x^{a}\right\}$ one can express the components of the affine connection completely in terms of the components of $g$ and $\sigma$ :

$\Gamma_{b c}^{a}=\left\{\begin{array}{l}a \\ b c\end{array}\right\}-\frac{1}{2} g^{a d}\left[g_{d b} \sigma_{c}+g_{d c} \sigma_{b}-g_{b c} \sigma_{d}\right]$

where $\left\{\begin{array}{l}a \\ b c\end{array}\right\}=\frac{1}{2} g^{a d}\left[g_{d b, c}+g_{d c, b}-g_{b c, d}\right]$ denotes the Christoffel symbols of second kind. The next proposition gives a helpful insight on the geometrical meaning of the Weyl parallel transport.

Corollary 2.1 Let $M$ be a differentiable manifold with an affine connection $\nabla$, a metric $g$ and a Weyl field of one-forms $\sigma$. If $\nabla$ is $W$-compatible, then for any smooth curve $\alpha=\alpha(\lambda)$ and any pair of two parallel vector fields $V$ and $U$ along $\alpha$, we have

$\frac{\mathrm{d}}{\mathrm{d} \lambda} g(V, U)=\sigma\left(\frac{\mathrm{d}}{\mathrm{d} \lambda}\right) g(V, U)$

where $\frac{\mathrm{d}}{\mathrm{d} \lambda}$ denotes the vector tangent to $\alpha$.

By integrating the above equation along the curve $\alpha$ from a point $p_{0}=\alpha\left(\lambda_{0}\right)$, we get

$g(V(\lambda), U(\lambda))=g\left(V\left(\lambda_{0}\right), U\left(\lambda_{0}\right)\right) e^{\int_{\lambda_{0}}^{\lambda} \sigma\left(\frac{\mathrm{d}}{\mathrm{d} \rho}\right) \mathrm{d} \rho}$.

Let us set $U=V$ and denote by $L(\lambda)$ the length of the vector $V(\lambda)$ at an arbitrary point $p=\alpha(\lambda)$ of the curve. It is easy to check that in a local coordinate system $\left\{x^{a}\right\}$ Eq. (5) reduces to

$\frac{\mathrm{d} L}{\mathrm{~d} \lambda}=\frac{\sigma_{a}}{2} \frac{\mathrm{d} x^{a}}{\mathrm{~d} \lambda} L$

Consider now the set of all closed curves $\alpha:[a, b] \in R \rightarrow$ $M$, i.e., with $\alpha(a)=\alpha(b)$. The equation

$g(V(b), U(b))=g(V(a), U(a)) e^{\int_{a}^{b} \sigma\left(\frac{\mathrm{d}}{\mathrm{d} \lambda}\right) \mathrm{d} \lambda}$

defines a holonomy group. If we want the elements of this group to correspond to an isometry, then we must require that

$\oint \sigma\left(\frac{d}{d \lambda}\right) d \lambda=0$ 
for any loop. From Stokes' theorem it follows that $\sigma$ must be an exact form, that is, there exists a scalar function $\phi$, such that $\sigma=\mathrm{d} \phi$. In this case we have a Weyl integrable manifold.

Weyl manifolds are completely characterized by the set $(M, g, \sigma)$, which will be called a Weyl frame. Let us remark that the Weyl compatibility condition (5) remains unchanged when we go to another Weyl frame $(M, \bar{g}, \bar{\sigma})$ by carrying out the following simultaneous transformations in $g$ and $\sigma$ :

$\bar{g}=e^{f} g$,

$\bar{\sigma}=\sigma+d f$,

where $f$ is an arbitrary scalar function defined on $M$. The conformal map (10) and the gauge transformation (11) define classes of equivalences in the set of Weyl frames. It is important to mention that the discovery that the compatibility condition (5) is invariant under this group of transformations was essential to Weyl's attempt at unifying gravity and electromagnetism, extending the concept of space-time to that of a collection of manifolds equipped with a conformal structure, leading to the notion that space-time might be viewed as a class $[g]$ of conformally equivalent Lorentzian metrics $[9-13,15,16]$.

\section{Weyl integrable space-time}

We now consider the particular case of a WIST, where $\sigma=$ $\mathrm{d} \phi$. As already mentioned, the set $(M, g, \phi)$ consisting of a differentiable manifold $M$ endowed with a metric $g$ and a Weyl scalar field $\phi$ will be referred to as a Weyl frame. In this case (11) becomes

$\bar{\phi}=\phi+f$.

If we set $f=-\phi$ in the above equation, we get $\bar{\phi}=0$. We refer to the set $\left(M, \bar{g}=e^{-f} g, \bar{\phi}=0\right)$ as the Riemannframe, because in this frame the manifold becomes Riemannian. On the other hand, it can easily be verified that Eq. (4) follows directly from $\nabla_{\alpha} \bar{g}_{\mu \nu}=0$. This result has interesting and useful consequences. In fact, the metric $\gamma=e^{-\phi} g$, defined for any frame $(M, g, \phi)$, is invariant under the Weyl transformations (10) and (11) any geometric quantity built exclusively with $\gamma$ is invariant. More generally, geometric objects such as the components of the curvature tensor $R_{\beta \mu \nu}^{\alpha}$, the components of the Ricci tensor $R_{\mu \nu}$, the scalar $e^{\phi} R$ are invariant under the Weyl transformations (10) and (11).

It is important to note that because the Weyl transformations (10) and (11) define an equivalence relation between frames $(M, g, \phi)$ it seems more appropriate to look into the equivalence class of such frames rather than on a particular frame. In other words, a Weyl manifold should be regarded as a frame $(M, g, \phi)$ that is only defined "up to a Weyl transformation". In this way, when dealing with a certain Weyl manifold we choose a particular frame in the equivalence class and consider that only geometric entities defined in that frame which are invariant are of interest, since they can be regarded as representative of the whole class. From this point of view, it is more natural to redefine some Riemannian concepts to meet the requirements of invariance. This procedure is analogous to the one adopted in conformal geometry, a branch of geometry, in which the geometric objects of interest are those invariant under conformal transformation, such as, say, the angle between two directions [32]. Accordingly, one should naturally generalize the definition of all invariant integrals when dealing with the integration of exterior forms. For example, the Riemannian $q$-dimensional volume form defined as $\Omega=\sqrt{-g} \mathrm{~d} x^{1} \wedge \cdots \wedge \mathrm{d} x^{q}$ is not invariant under Weyl transformations, hence it should be replaced by $\Omega=\sqrt{-\gamma} e^{-\frac{q}{2} \phi} \mathrm{d} x^{1} \wedge \cdots \wedge \mathrm{d} x^{q}$, and so on. ${ }^{1}$ Likewise, in a Weyl integrable manifold it is more natural to define the concept of "length of a curve" in an invariant way. It follows that our familiar notion of proper time as the arc length of worldlines in four-dimensional Lorentzian space-time should be modified. Because of this, we shall redefine the proper time $\Delta \tau$ measured by a clock moving along a parametrized timelike curve $x^{\mu}=x^{\mu}(\lambda)$ between $x^{\mu}(a)$ and $x^{\mu}(b)$, in such a way, that $\Delta \tau$ is the same in all frames. This suggests the following definition:

$$
\begin{aligned}
\Delta \tau & =\int_{a}^{b}\left(\bar{g}_{\mu \nu} \frac{\mathrm{d} x^{\mu}}{\mathrm{d} \lambda} \frac{\mathrm{d} x^{\nu}}{\mathrm{d} \lambda}\right)^{\frac{1}{2}} \mathrm{~d} \lambda \\
& =\int_{a}^{b} e^{-\frac{\phi}{2}}\left(g_{\mu \nu} \frac{\mathrm{d} x^{\mu}}{\mathrm{d} \lambda} \frac{\mathrm{d} x^{\nu}}{\mathrm{d} \lambda}\right)^{\frac{1}{2}} \mathrm{~d} \lambda
\end{aligned}
$$

It must be noted that the above expression can also be obtained from the special relativistic definition of proper time if we adopt the prescription $\eta_{\mu \nu} \rightarrow e^{-\phi} g_{\mu \nu}$. It is clear that the right-hand side of this equation is invariant under Weyl transformations and that, in the Riemann frame, it reduces to the definition of proper time in general relativity. We, therefore, take $\Delta \tau$, as given above, as the extension to an arbitrary Weyl frame of general relativistic clock hypothesis, i.e., the assumption that $\Delta \tau$ measures the proper time measured by a clock attached to the particle.

It is now easy to see that the extremization of the functional (13) leads to the equations

$$
\begin{aligned}
& \frac{\mathrm{d}^{2} x^{\mu}}{\mathrm{d} \lambda^{2}}+\left(\left\{\begin{array}{l}
\mu \\
\alpha \beta
\end{array}\right\}-\frac{1}{2} g^{\mu \nu}\left(g_{\alpha \nu} \phi_{, \beta}+g_{\beta \nu} \phi_{, \alpha}-g_{\alpha \beta} \phi, \nu\right)\right) \\
& \times \frac{\mathrm{d} x^{\alpha}}{\mathrm{d} \lambda} \frac{\mathrm{d} x^{\beta}}{\mathrm{d} \lambda}=0
\end{aligned}
$$

\footnotetext{
${ }^{1}$ Note that $g$ in the expression $\sqrt{-g}$ denotes the determinant of $g_{\mu \nu}$.
} 
where $\left\{\begin{array}{l}\mu \\ \alpha \beta\end{array}\right\}$ designates the Christoffel symbols calculated with $g_{\mu \nu}$. Recall that in the derivation of the above equations the parameter $\lambda$ must be chosen such that

$e^{-\phi} g_{\alpha \beta} \frac{\mathrm{d} x^{\alpha}}{\mathrm{d} \lambda} \frac{\mathrm{d} x^{\beta}}{\mathrm{d} \lambda}=K=\mathrm{const}$

along the curve, which, up to an affine transformation, permits us to identify $\lambda$ with the proper time $\tau$. Surely, these equations are exactly those that yield the affine geodesics in a Weyl integrable space-time, since they can be rewritten as

$\frac{\mathrm{d}^{2} x^{\mu}}{\mathrm{d} \tau^{2}}+\Gamma_{\alpha \beta}^{\mu} \frac{\mathrm{d} x^{\alpha}}{\mathrm{d} \tau} \frac{\mathrm{d} x^{\beta}}{\mathrm{d} \tau}=0$,

where $\Gamma_{\alpha \beta}^{\mu}=\left\{\begin{array}{l}\mu \\ \alpha \beta\end{array}\right\}-\frac{1}{2} g^{\mu \nu}\left(g_{\alpha \nu} \phi_{, \beta}+g_{\beta \nu} \phi_{, \alpha}-g_{\alpha \beta} \phi, v\right)$, according to (4), are identified with the components of the Weyl connection. Thus the extension of the geodesic postulate by requiring that the functional (13) be an extremum is equivalent to assuming that the particle motion must follow affine geodesics defined by the Weyl connection $\Gamma_{\alpha \beta}^{\mu}$. Let us note that, as a consequence of the Weyl compatibility condition (3) between the connection and the metric, Eq. (15) holds automatically along any affine geodesic determined by (16). Since both the connection components $\Gamma_{\alpha \beta}^{\mu}$ and the proper time $\tau$ are invariant when we switch from one Weyl frame to the other, Eq. (16) are invariant under Weyl transformations.

As is well known, the geodesic postulate is not only concerned with the motion of particles, but it also determines the propagation of light rays in space-time. On the other hand, since the paths of light rays are null curves, one cannot use the proper time as a parameter to describe these curves. Thus light rays are supposed to follow null affine geodesics, which cannot be defined in terms of the functional (13), but, instead, they must be characterized by their behavior with respect to parallel transport. We naturally extend this postulate by simply assuming that light rays follow Weyl null affine geodesics.

\section{The Raychaudhuri equation}

The Raychaudhuri equation played a fundamental role in the derivation of the Hawking-Penrose singularity theorems. In the derivation, however, it is assumed right from the beginning that the geometry underlying the space-time is Riemannian. In this section, we investigate the extension of this equation to the case of a Weyl integrable space-time.

Let us first remark that the extension of the geodesic postulate to WIST assumes that the particle motion must follow Weyl time-like geodesics. In the following we shall consider a smooth congruence $\Gamma$ of time-like geodesics corresponding to the worldlines of a class of observers, parametrized by the invariant proper time $\tau$ defined in (13). Hence, the tangents to the congruence generate a tangent vector field $V$ normalized to unit length. In order to keep the formalism invariant under Weyl transformations we shall choose the affine parameter of the congruence as the Weyl invariant arc length, i.e., we normalize $V$ with respect to the invariant metric $\gamma_{\mu \nu}=e^{-\phi} g_{\mu \nu}$,

$\gamma(V(\tau), V(\tau))=1$

Therefore, in a local coordinate system, a geodesic curve described by $x^{\mu}(\tau)$ satisfies $V^{\mu} \nabla_{\mu} V^{\alpha}=0$, where $V^{\alpha}=\frac{\mathrm{d} x^{\alpha}}{\mathrm{d} \tau}$ and we have the affine geodesics in a Weyl integrable spacetime shown in (16). Furthermore, once we have normalized the tangent vector field with the invariant metric $\gamma_{\mu \nu}$, we obtain

$V_{\mu} \nabla_{\alpha} V^{\mu}=0=V^{v} \nabla_{\alpha} V_{\nu}$

since $\nabla_{\alpha} \gamma_{\mu \nu}=0$.

Let us now consider, at some point $p$ of $M$, the hypersurface $\Sigma$ orthogonal to the vector field $V$. As in the standard procedure, we define the operator of projection $\Pi$ onto the hypersurface $\Sigma$ as

$\Pi_{\mu \nu}=\gamma_{\mu \nu}-V_{\mu} V_{\nu}$

As is well known, $\Pi_{\mu \nu}$ represents to the first fundamental form of the hypersurface induced by the metric $\gamma$ and its role is to project any vector of $T_{p} M$ at $p$ onto $T_{p} \Sigma$, the tangent space to the submanifold $\Sigma .^{2}$

We proceed with the derivation of the Raychaudhuri equation in this new setting. We first need to consider a smooth one-parameter subfamily $\alpha_{S}(\tau)$ of geodesics in the congruence of $V$ and then define a deviation vector $\eta$ that represents an infinitesimal spatial displacement from a given geodesic $\alpha_{o}(\tau)$ to a neighboring geodesic in this subfamily. Once we have been given $\eta$ in $\Sigma$, we define the vector field $\eta$ along this subfamily by Lie dragging it along $V$, that is, by requiring that

$Ł_{V}(\eta)=0$.

From the definition of Lie derivative and the fact that the connection $\nabla$ is assumed to be torsionless we are led to the following equation:

$V^{\mu} \nabla_{\mu} \eta^{\alpha}=\eta^{\mu} \nabla_{\mu} V^{\alpha}$

\footnotetext{
2 Note that we are using the invariant metric $\gamma$ in order to ensure a invariant projection tensor. Accordingly, the operation of raising and lowering tensorial indices must be always carried out with $\gamma$. This guarantees that the duality between covariant and contravariant vectors is not modified by Weyl transformations.
} 
which shows how the deviation vector changes along the congruence. An important role played in the investigation of the behavior of neighboring geodesics as we go along the congruence $\Gamma$ is played by the so-called deformation tensor $Q_{\mu \nu}$, defined as

$Q_{\mu \nu}=\nabla_{\nu} V_{\mu}$.

In terms of $Q_{\mu \nu}$ we can rewrite (21) as

$V^{\mu} \nabla_{\mu} \eta^{\alpha} \equiv Q_{\mu}^{\alpha} \eta^{\mu}$

which clearly means that the deformation tensor measures the failure of $\eta^{\mu}$ to be parallel transported [33]. Furthermore, it is easy to see that this tensor is purely spatial, since $Q_{\mu \nu} V^{\mu}=$ $0=Q_{\mu \nu} V^{\nu}$. Thus, $Q_{\mu \nu}$ is a tensor defined in the subspace of the tangent space perpendicular to $V$. Finally, in order to have a physical interpretation of some kinematical aspects of the congruence $\Gamma$, we can decompose $Q_{\mu \nu}$ in its irreducible parts:

$Q_{\mu \nu} \equiv \frac{1}{3} \Theta \Pi_{\mu \nu}+\sigma_{\mu \nu}+\omega_{\mu \nu}$,

where the parameters $\Theta, \sigma_{\mu \nu}$, and $\omega_{\mu \nu}$ are known, respectively, as the expansion, shear and vorticity of the congruence (for instance, Ref. [33]). From the above equation we have

$\Theta=\Pi^{\mu \nu} Q_{\mu \nu}$,

$\sigma_{\mu \nu}=Q_{(\mu \nu)}-\frac{1}{3} \Theta \Pi_{\mu \nu}$,

$\omega_{\mu \nu}=Q_{[\mu \nu]}$.

It is easy to see that in the case where the congruence is locally orthogonal to the hypersurface $\Sigma$, we have $\omega_{\mu \nu}=0$.

We are now particularly interested in the behavior of $\Theta$, which measures the expansion of the congruence and can tell us about the existence of conjugated points. Thus, in order to obtain the Raychaudhuri equation, we need to know the rate of change of $\Theta$ along the congruence. Therefore, since, by definition, $\Theta=\nabla_{\alpha} V^{\alpha}$, we must compute $V^{\mu} \nabla_{\mu} \Theta=$ $V^{\mu} \nabla_{\mu}\left[\nabla_{\alpha} V^{\alpha}\right]$. On the other hand, from the definition of the curvature tensor, we have

$\nabla_{\beta} \nabla_{\nu} V^{\mu}-\nabla_{\nu} \nabla_{\beta} V^{\mu}=R_{\lambda \nu \beta}^{\mu} V^{\lambda}$

where

$R_{\lambda \nu \beta}^{\mu}=\partial_{\beta} \Gamma_{\lambda \nu}^{\mu}-\partial_{\nu} \Gamma_{\lambda \beta}^{\mu}+\Gamma_{\lambda \nu}^{\rho} \Gamma_{\rho \beta}^{\mu}-\Gamma_{\lambda \beta}^{\rho} \Gamma_{\rho \nu}^{\mu}$,

one can readily obtain

$V^{\mu} \nabla_{\mu} \Theta=-\frac{1}{3} \Theta^{2}-2\left(\sigma^{2}-\omega^{2}\right)+R_{\mu \nu} V^{\mu} V^{\nu}$, where we denote $\sigma^{2}=\sigma_{\mu \nu} \sigma^{\mu \nu}, \omega^{2}=\omega_{\mu \nu} \omega^{\mu \nu}$, and the term $R_{\mu \nu} V^{\mu} V^{\nu}$ is usually referred to as the Raychaudhuri scalar. Setting $\dot{\Theta}=V^{\mu} \nabla_{\mu} \Theta$, we can write (30) in the following form, known as the Raychaudhuri equation:

$\dot{\Theta}+\frac{1}{3} \Theta^{2}+2\left(\sigma^{2}-\omega^{2}\right)=R_{\mu \nu} V^{\mu} V^{\nu}$.

It is worth noting that (31) has the same form as in the case of a Riemannian space-time, although it must be recalled that the Ricci tensor is built with the Weylian connection. In fact, this is not surprising as we have redefined the proper time in an invariant way, using the invariant metric $\gamma_{\mu \nu}=e^{-\phi} g_{\mu \nu}$. In the next section, we shall analyze the conditions that lead to singularities in the space-time.

\section{Extending the singularity theorem}

Because of the form of the Raychaudhuri equation takes in a Weyl integrable space-time, the description of conjugate points is the same as in Riemannian geometry. Thus, we have the following statement: if $\theta_{0}=\theta\left(\tau_{0}\right)<0$ for some $\tau=\tau_{0}, R_{\mu \nu} V^{\mu} V^{\nu} \leq 0$, then in a finite invariant proper time $\tau \leq 3 /\left|\theta_{0}\right|$, the congruence will develop a conjugate point $\theta \rightarrow-\infty$. As a matter of fact, the extension of any theorem from Riemannian geometry to a Weyl integrable can be trivially carried out by simpling considering the invariant metric $\gamma=e^{-\phi} g$. For instance, as we have already mentioned the extremization of the functional (13) leads directly to the Weylian geodesics. On the other hand, results coming from differential topology and concerning the causal structure of space-time that are valid in a Riemannian space-time are also valid in a Weyl integrable space-time since conformal transformations do not affect the light-cone structure nor the manifold orientability. Indeed, the proof of one of the most important results we are going to enunciate now, namely, the generalized Jacobi theorem, proceeds along the same lines of reasoning employed in the Riemannian case, where we merely replace $g$ by the invariant metric $\gamma=e^{-\phi} g$ [33].

Theorem 5.1 (Jacobi) Let $\gamma:[0,1] \rightarrow M$ a differential time-like curve connecting points $p, q \in M$. Then a necessary and sufficient condition for $\gamma$ to locally maximize the invariant arc length between $p$ and $q$ is that $\gamma$ be a Weyl geodesic without conjugate points between $p$ and $q$.

Note the relation between the Raychaudhuri scalar $\Theta$ and the extrinsic curvature of the submanifold orthogonal to geodesic congruence, which is represented by the mixed tensor $Q^{\mu}{ }_{v}=\Pi_{\alpha}^{\mu} \Pi^{\beta}{ }_{v} \nabla_{\beta} V^{\alpha}$. For the case of a congruence of geodesics orthogonal to hypersurface $\Sigma$ and parameterized by the invariant arc length we have $Q_{\nu}^{\mu}=\nabla_{\nu} V^{\mu}$. Thus, the trace of $Q_{\nu}^{\mu}$ is equal to $\Theta$, i.e., $Q=Q_{\alpha}^{\alpha}=\Theta$. Now we are ready to prove the following proposition. 
Theorem 5.2 Let $(M, g, \phi)$ be a globally hyperbolic Weylian space-time with $R_{\mu \nu} V^{\mu} V^{\nu} \leq 0$ for all time-like vectors $V$. Suppose there exists a space-like Cauchy surface $\Sigma$ such that the trace of its extrinsic curvature (for the orthonormal congruence of past directed geodesics) satisfies $B \leq C<0$ over the whole surface, $B$ and $C$ being constants. Then no past directed time-like curve coming from $\Sigma$ can have an invariant arc length greater than $3 /|C|$. In particular, all past directed time-like geodesics are incomplete.

Proof Let us prove this theorem by contradiction. Suppose there exists a time-like curve $\lambda=\lambda(\tau)$ coming from $\Sigma$ whose value of the invariant arc length $\tau$ at some point $p$ $\in \lambda$ is greater than $3 /|C|$. It is well known that as the set of curves joining two points in a globally hyperbolic manifold is compact, the invariant arc length function must have a maximum value for a given curve. Then this curve must be a Weyl geodesic. Therefore, there must be a geodesic $\gamma$ (with invariant arc length greater than $3 /|C|$ ) joining $p$ to $\Sigma$. This means there are no conjugated points between $p$ and $\Sigma$. But, from Raychaudhuri's inequality, we know that $\gamma$ must have conjugated points between $p$ and $\Sigma$, which is a contradiction. We then conclude that the original curve $\lambda$ cannot exist.

In the case of general relativity where the space-time mathematical structure is that of Riemannian geometry, which is a special case of Weyl geometry when $\phi$ is a constant of motion, the geometric condition

$R_{\mu \nu} V^{\mu} V^{\nu} \leq 0$

is equivalent to the so-called strong energy condition

$T_{\mu \nu} V^{\mu \nu}-T / 2 \geq 0$,

and requiring that $Q<0$ is equivalent to assuming that in the course of the cosmic evolution the Universe underwent an expansion period, which seems to be a rather reasonable assumption. In view of the above, this leads to the conclusion that the Universe, as modeled by GR, must necessarily have had a beginning starting from a singular state. Let us now consider some different scenarios offered by two alternative gravitational theories, namely, the Novello-Oliveira-SalimElbaz's theory (N) [17,34] and a recent geometrical approach to scalar-tensor theory (GST), both inspired by the idea that space-time can be described by Weyl integrable geometry [35].

\subsection{Novello's theory}

Novello's theory starts with the action

$S=\int \mathrm{d}^{4} x \sqrt{-g}\left\{R-2 \xi \phi^{, \mu} \phi_{, \mu}+e^{-2 \phi} L_{m}\right\}$, where $R$ denotes the Weylian scalar curvature, $\phi$ represents the Weyl scalar field, $\xi$ is a dimensionless parameter, while $L_{m}$ stands for the Lagrangian of the matter fields. The form of $L_{m}$ is determined from the corresponding Lagrangian in special relativity by replacing the ordinary derivatives by covariant derivatives with respect to the Weyl connection. The field equations are given by

$$
\begin{aligned}
& R_{\mu \nu}-\frac{1}{2} g_{\mu \nu}=-\nabla_{\mu} \phi_{, \nu} \\
& \quad+(2 \xi-1) \phi_{, \mu} \phi_{, \nu}-\xi \phi_{, \alpha} \phi^{, \alpha} g_{\mu \nu}-e^{-\phi} T_{\mu \nu}, \\
& \nabla_{\alpha} \phi^{, \alpha}+2 \phi_{, \alpha} \phi^{, \alpha}=-\frac{1}{2 \lambda} e^{-\phi} T,
\end{aligned}
$$

where we have set $\lambda=\frac{3}{2}-2 \xi$.

From the above equations it is not difficult to verify that

$$
\begin{aligned}
R_{\mu \nu} V^{\mu} V^{\nu}= & -\frac{\mathrm{d}^{2} \phi}{\mathrm{d} \bar{s}^{2}}+(2 \xi-1)\left(\frac{\mathrm{d} \phi}{\mathrm{d} \bar{s}}\right)^{2} \\
& -e^{-\phi}\left(T_{\mu \nu} V^{\mu} V^{\nu}-\frac{1}{2}|V|^{2} T+\frac{1}{4 \lambda}|V|^{2} T\right) .
\end{aligned}
$$

Clearly, in this theory (32) does not imply the violation of the strong energy condition (33), but rather we have the following situation. Consider the conditions below:

$\frac{\mathrm{d}^{2} \phi}{\mathrm{d} \bar{s}^{2}} \equiv \ddot{\phi} \geq 0$,
$2 \xi-1 \leq 0$,

$T_{\mu \nu} V^{\mu} V^{\nu}-\frac{1}{2}|V|^{2} T+\frac{1}{4 \lambda}|V|^{2} T \geq 0$.

If any one of these conditions is violated, then the solution may correspond to a non-singular space-time. Consider, as an example, the vacuum solution of the field equations (35) and (36) obtained in Ref. [34]. By assuming homogeneity and isotropy we can write

$\mathrm{d} s^{2}=\mathrm{d} t^{2}-a^{2}(t)\left(\frac{\mathrm{d} r^{2}}{1-\kappa r^{2}}+r^{2} \mathrm{~d} \Omega^{2}\right)$,

$\phi_{, \alpha}=\phi_{, 0} \delta_{\alpha}^{0} \doteq \dot{\phi} \delta_{\alpha}^{0}$.

This leads to the equations

$\dot{a}^{2}+\kappa+\frac{(4 \xi-3)}{6}(\dot{\phi} a)^{2}=0$,

$2 a \ddot{a}+\dot{a}^{2}+\kappa-\frac{4 \xi-3}{4}(\dot{\phi} a)^{2}=0$,

$\left(a^{3} \dot{\phi}\right)_{, 0}=0$.

By integrating the last of these equations we get $\dot{\phi}=\zeta a^{-3}$, where $\zeta$ is a constant. Now, if $4 \xi-3>0$, it follows that

$\dot{a}^{2}(t)=1-\left[\frac{a_{0}}{a(t)}\right]^{4}$, 
with $\left(a_{0}\right)^{4}=\left(\frac{4 \xi-3}{12}\right) \zeta^{2}$, thus implying that $a(t) \geq a_{0}$. The model describes a non-singular bouncing universe, a scenario in which after undergoing a period of contraction, dominated by the scalar field, the universe reaches a minimum value, and then starts to expand, at an inflationary rate, until the radiation dominates over the scalar field and the scale factor begins to follow the standard cosmic evolution [34]. Clearly, the non-singular behavior of the model is a consequence of the fact that the condition $\xi \leq 1 / 2$ is violated. In order to display the bouncing character of this model, it is convenient to solve (46) in terms of the conformal time $\eta$, defined as $\mathrm{d} \eta=\mathrm{d} t / a(t)$. It can be shown that the scale factor and the scalar field are given, respectively, by

$$
\begin{aligned}
& a(\eta)=a_{0} \sqrt{\cosh 2\left(\eta-\eta_{0}\right)}, \\
& \phi(\eta)=\frac{\zeta}{2 a_{0}^{2}} \arccos \left[\cosh 2\left(\eta-\eta_{0}\right)\right]^{-1} .
\end{aligned}
$$

\subsection{Geometrical scalar-tensor theory}

The geometrical approach to scalar-tensor theory starts with the Brans-Dicke action

$$
S=\int \mathrm{d}^{4} x \sqrt{-g} e^{-\phi}\left[R+\omega \phi^{, \alpha} \phi_{, \alpha}+k^{*} e^{-\phi} L_{m}\left(e^{-\phi} g\right)\right],
$$

where $\phi$ is assumed a priori to be a geometrical field, i.e., an intrinsic part of the space-time geometry, $\omega$ is a dimensionless parameter and $k^{*}=\frac{8 \pi}{c^{4}}$. By applying the Palatini variational method, one obtains the Weyl integral compatibility condition [35],

$$
\nabla_{\alpha} g_{\mu \nu}=g_{\mu \nu} \phi_{, \alpha}
$$

Naturally, the action (49) can easily be extended to accommodate a scalar potential $\mathcal{V}(\phi)$ and to allow for a functional dependence of $\omega$ on $\phi$, thus leading to [36]

$$
\begin{aligned}
S= & \int \mathrm{d}^{4} x \sqrt{-g}\left\{e^{-\phi}\left[R+\omega(\phi) g^{\mu \nu} \phi_{, \mu} \phi, \nu\right]\right. \\
& \left.-\mathcal{V}(\phi)+\kappa^{*} e^{-2 \phi} L_{m}\right\} .
\end{aligned}
$$

The field equations obtained from (49) are given by

$$
\begin{gathered}
R_{\mu \nu}-\frac{1}{2} g_{\mu \nu} R=\omega(\phi)\left(\frac{1}{2} g_{\mu \nu} \phi_{, \alpha} \phi^{, \alpha}-\phi_{, \mu} \phi, \nu\right) \\
-\frac{1}{2} e^{\phi} g_{\mu \nu} \mathcal{V}(\phi)-\kappa^{*} T_{\mu \nu}, \\
\square \phi=-\left(1+\frac{1}{2 \omega} \frac{\mathrm{d} \omega}{\mathrm{d} \phi}\right) \phi_{, \alpha} \phi^{, \alpha}-\frac{e^{\phi}}{\omega}\left(\frac{1}{2} \frac{\mathrm{d} \mathcal{V}}{\mathrm{d} \phi}+\mathcal{V}\right),
\end{gathered}
$$

where $\square$ denotes the d'Alembertian operator calculated with the Weyl connection.
By taking into account (52) one easily obtains

$$
\begin{aligned}
R_{\mu \nu} V^{\mu} V^{\nu} \equiv & -\kappa^{*}\left(T_{\mu \nu} V^{\mu} V^{\nu}-\frac{1}{2}|V|^{2} T\right) \\
& -\omega(\phi) \dot{\phi}^{2}+|V|^{2} e^{\phi} \mathcal{V}(\phi) .
\end{aligned}
$$

As we can see, here again the (32) does not require the violation of the strong energy condition (33). Thus, if we assume that (33) holds, then any space-time described by these equations will satisfy $R_{\mu \nu} V^{\mu} V^{\nu} \leq 0$, as long as

$\omega(\phi) \dot{\phi}^{2}-e^{\phi}|V|^{2} \mathcal{V}(\phi) \geq 0$.

We conclude that in this case the singularity theorem applies. On the other hand, if (55) is violated, then the solution may correspond to a non-singular space-time.

In the following let us consider some solutions to $(52,53)$, for some choices of the potential $\mathcal{V}(\phi)$ in the case $\omega(\phi)=$ constant and $T_{\mu \nu}=0$. These solutions correspond to homogeneous and isotropic models in GST theory and are obtained in the Riemann frame $\left(M, \bar{g}=e^{-f} g, \bar{\phi}=0\right)$, in which case $|V|^{2}=e^{\phi}$. If we take the line element written as in (42), then the field equations (52) reduce to

$3 \frac{\dot{a}^{2}}{a^{2}}+3 \frac{\epsilon}{a^{2}}=\frac{\omega}{2} \dot{\phi}^{2}+\frac{e^{2 \phi}}{2} \mathcal{V}(\phi)$,

$2 \frac{\ddot{a}}{a}+\left(\frac{\dot{a}}{a}\right)^{2}+\frac{\epsilon}{a^{2}}=-\frac{\omega}{2} \dot{\phi}^{2}+\frac{e^{2 \phi}}{2} \mathcal{V}(\phi)$,

while (53) gives

$\ddot{\phi}+3 \frac{\dot{a}}{a} \dot{\phi}=-\frac{e^{2 \phi}}{\omega}\left(\mathcal{V}(\phi)+\frac{1}{2} \frac{\mathrm{d} \mathcal{V}}{\mathrm{d} \phi}\right)$,

where $\epsilon=0, \pm 1$, according to the curvature of the spatial section. $^{3}$

Solutions to Eqs. (56)-(58) with flat spatial section $\epsilon=0$ were obtained for the following choices of the scalar potential: $e^{-2 \phi} \Lambda, e^{-(2+\lambda) \phi} \mathcal{V}_{o}, e^{-2 \phi}\left(m \phi^{2}+\Lambda\right)$, and $2 \lambda e^{-2 \phi}\left(\phi^{2}+\right.$ $2 \omega / 3)^{2}$, where $\Lambda, \mathcal{V}_{o}, \lambda$, and $m$ are constants. These, in the Riemann frame, correspond to the cosmological constant, the dilaton field, the massive scalar field, and a field with a quartic interaction. These three cases are displayed in the tables below, where the presence of a singular behavior is determined according to whether or not (55) holds (Table 1).

3 It is interesting to note here that from the above we can obtain the following equation:

$\dot{H}=-\frac{\omega}{2} \dot{\phi}^{2}+\frac{\epsilon}{a^{2}}$.

This equation might be useful to set the possible values of $\omega$. 
Table 1 The other parameters $\left\{\mathcal{V}_{o}, \lambda, m, \Lambda\right.$, and $\left.\phi_{o}\right\}$ are constants

\begin{tabular}{lll}
\hline Potential & Solution to $\phi(t)$ & Where \\
\hline$e^{-2 \phi} \Lambda$ & $\phi(t)=\phi_{o} \pm \sqrt{\frac{2}{3|\omega|}} \arctan \left[\sinh \left(\frac{\sqrt{6 \Lambda}}{2}\left(t-t_{0}\right)\right)\right]$ & $\omega<0$ and $\Lambda>0$ \\
$e^{-(\lambda+2) \phi} \mathcal{V}_{o}$ & $\phi(t)=\frac{2}{\lambda} \ln \left(\frac{H_{0} \lambda^{2}}{2 \omega} t+e^{\frac{\lambda}{2} \phi_{o}}\right)$ & $H_{o}= \pm \sqrt{\frac{\omega \mathcal{V}_{o}}{6 \omega-\lambda^{2}}}$ \\
$e^{-2 \phi}\left(m \phi^{2}+\Lambda\right)$ & $\phi(t)=\phi_{o}-\frac{2 \alpha}{\omega} t$ & $\alpha= \pm \sqrt{-\frac{\omega \Lambda}{4}}$ \\
$e^{-2 \phi} 2 \lambda\left(\phi^{2}-2 / 3 \omega\right)^{2}$ & $\phi(t)=\phi_{o} \exp \left(-\frac{4 A}{\omega} t\right)$ & $A= \pm \sqrt{\frac{\lambda}{3}}$ \\
\hline
\end{tabular}

Table 2 The parameters are the same as in Table 1 and $a_{o}$ is also a constant

\begin{tabular}{lll}
\hline Potential & Solution to $a(t)$ & Singularity in finite time \\
\hline$e^{-2 \phi} \Lambda$ & $a(t)=a_{0} \cosh \left[\frac{\sqrt{6 \Lambda}}{2}\left(t-t_{0}\right)\right]^{1 / 3}$ & None \\
$e^{-(\lambda+2) \phi} \mathcal{V}_{o}$ & $a(t)=a_{o}\left[\frac{\lambda^{2} H_{o}}{2 \omega} e^{-\frac{\lambda}{2} \phi_{o}} t+1\right]^{2 \omega / \lambda^{2}}$ & Singular if $\omega>\lambda^{2} / 6$ \\
$e^{-2 \phi}\left(m \phi^{2}+\Lambda\right)$ & $a(t)=a_{o} \exp \left[\alpha t\left(\phi_{o}-\frac{\alpha}{\omega} t\right)\right]$ & Non-singular if $\omega<0$ \\
$e^{-2 \phi} 2 \lambda\left(\phi^{2}-\frac{2}{3 \omega}\right)^{2}$ & $a(t)=a_{o} \exp \left[-\frac{\omega \phi_{o}^{2}}{8} \exp \left(-\frac{8 A}{\omega} t-1\right)+\frac{2}{3 \omega} A t\right]$ & Singular if $\omega>0$ \\
\hline
\end{tabular}

\section{Final remarks}

The Hawking-Penrose singularity theorems are a direct consequence of Einstein's theory of gravity. Given the important role they have played in our understanding of the Universe, as modeled by general relativity, it is also of interest to find the analogous of these theorems in alternative gravity theories. Singularity theorems and energy conditions have been studied in connection with Brans-Dicke theory, perhaps the most popular scalar-tensor gravity theories. However, their meaning remains still controversial due to the question of whether or not the Einstein frame and the Jordan frame are physically equivalent $[37,38]$. In the present geometrical approach, this controversy does not arise, as the physical entities defined in the theory are naturally invariant under frame transformations [39-42] (Table 2).

Finally, it has been shown that Weyl integral space-time seems to be the natural geometrical scenario that arises in the context of scalar-tensor theories, at least, whenever one has a theory that assumes a non-minimal coupling of the scalar field to the Ricci curvature [35]. The basic principle which underlies the determination of the space-time geometry would be, in this case, the adoption of the Palatini variational principle, which has played an important role in recently proposed modified theories of gravity [43]. The investigation of the behavior of space-time singularities in this new framework was one of our main motivations to write the present paper.

Acknowledgments The authors thanks CNPq/CAPES for financial support. I. P. Lobo is supported by the CAPES-ICRANet Program (BEX $14632 / 13-6)$
Open Access This article is distributed under the terms of the Creative Commons Attribution 4.0 International License (http://creativecomm ons.org/licenses/by/4.0/), which permits unrestricted use, distribution, and reproduction in any medium, provided you give appropriate credit to the original author(s) and the source, provide a link to the Creative Commons license, and indicate if changes were made. Funded by $\mathrm{SCOAP}^{3}$.

\section{References}

1. E.M. Lifshitz, V.V. Sudakov, I.M. Khalatinikov, Sov. Phys. JETP 13, 1298 (1961)

2. R. Penrose, Phys. Rev. Lett. 14, 57-59 (1965)

3. S.W. Hawking, R. Penrose, Proc. R. Soc. Lond. A 314, 529 (1970)

4. S.W. Hawking, G.F.R. Ellis, The Large Scale Structure of SpaceTime (Cambridge University Press, Cambridge, 1973)

5. J.M.M. Senovilla, D. Garfinkle, Class. Quantum Gravity 32, 124008 (2015)

6. M. Novello, J.M. Salim, F.T. Falciano, Int. J. Geom. Methods Mod. Phys. 8, 87-98 (2011)

7. H.F.M. Gönner, Living Rev. Relativ. 7, 2 (2004)

8. H.F.M. Gönner, Living Rev. Relativ. 17, 5 (2014)

9. H. Weyl, Sitzungesber Deutsch. Akad. Wiss. Berl. 465 (1918)

10. H. Weyl, Space, Time, Matter (Dover, New York, 1952)

11. A nice account of Weyl's ideas as well as the refutation of his gravitational theory may be found in W. Pauli, Theory of Relativity (Dover, New York, 1981)

12. L. O'Raiefeartaigh, N. Straumann, Rev. Mod. Phys. 72, 1 (2000)

13. N. Rosen, Found. Phys.12, 213 (1982)

14. For a more formal mathematical treatment, see G.B. Folland, J. Differ. Geom. 4, 145 (1970)

15. For a comprehensive review on Weyl geometry see E. Scholz. arXiv:1111.3220 [math.HO]

16. E. Scholz. arXiv:1206.1559

17. M. Novello, H. Heintzmann, Phys. Lett. A 98, 10 (1983)

18. For gravitational theories in general formulated in WIST and related topics, see K.A. Bronnikov, Yu.M. Konstantinov, V.N. Melnikov, Graviy. Cosmol. 1, 60 (1995) 
19. J.M. Salim, S.L. Sautï, Class. Quantum Graity 13, 353 (1996)

20. H.P. de Oliveira, J.M. Salim, S.L. Sautï, Class. Quantum Gravity 14, 2833 (1997)

21. V. Melnikov, Classical Solutions in Multidimensional Cosmology in Proceedings of the VIII Brazilian School of Cosmology and Gravitation II, ed. by M. Novello (Editions Frontiïres, 1995), p. 542

22. R.G. Gannouji, H. Nandan, N. Dadhich, JCAP 11, 51 (2011)

23. O. Arias, R. Cardenas, I. Quiros. Nucl. Phys. B 643, 187 (2002)

24. J. Miritzis, Class. Quantum Gravity 21, 3043 (2004)

25. J. Miritzis, J. Phys. Conf. Ser. 8, 131 (2005)

26. M. Israelit, Found. Phys. 35, 1725 (2005)

27. J.E. Madriz Aguilar, C. Romero, Found. Phys. 39, 1205 (2009)

28. T. Moon, J. Lee, P. Oh, Mod. Phys. Lett. A 25, 3129 (2010). arXiv:0912.0432 [gr-qc]

29. C. Romero, J.B. Fonseca-Neto, M.L. Pucheu, Class. Quantum Gravity 29, 155015 (2012)

30. M.P. do Carmo, Riemannian Geometry (Birkhauser, Boston, 1991)

31. F. Dahia, G.A.T. Gomez, C. Romero, J. Math. Phys. 49, 102501 (2008)

32. S. Kobayashi, Transformation Groups in Differential Geometry (Springer, New York, 1972)

33. R.M. Wald, General Relativity (The University of Chicago Press, Chicago, 1984)
34. M. Novello, L.A.R. Oliveira, J.M. Salim, E. Elbas, Int. J. Mod. Phys. D 1, 641 (1992)

35. T.S. Almeida, M.L. Pucheu, C. Romero, J.B. Formiga, Phys. Rev. D 89, 064047 (2014)

36. M.L. Pucheu, F.A.P. Alves Júnior, C. Romero, Cosmological Models in Geometrical Scalar-Tensor Theories (in preparation)

37. V. Faraoni, Cosmology in Scalar-Tensor Gravity (Kluwer Academic Publishers, Dordrecht, 2004)

38. A. Bhadra, K. Sarkar, D.P. Datta, K.K. Nandi, Mod. Phys. Lett. A 22, 367 (2007)

39. For other frame-invariant approaches to scalar-tensor theories, see R. Catena, M. Pietroni, L. Scaralbello, Phys. Rev. D 76, 084039 (2007)

40. L. Jïr, P. Kuusk, M. Saal, O. Vilson, Phys. Rev. D 91, 024041 (2015)

41. I. Quiros, R. Garcia-Salcedo, J.E.M. Aguilar, T. Matos, Gen. Relativ. Gravit. 45, 489 (2013)

42. E. Scholz. arXiv: 1111.3220

43. V. Faraoni, S. Capozziello, A Survey of Gravitational Theories for Cosmology and Astrophysics (Springer, New York, 2010) 